\title{
含噁二唑杨梅素衍生物的合成及生物活性研究
}

\author{
张橙蒋仕春陈英郭涛夏榕娇 \\ 汤旭陈丽娟贺鸣薛伟*
}

(绿色农药与农业生物工程国家重点实验室培育基地 教育部绿色农药与农业生物工程重点实验室

贵州大学精细化工研究开发中心 贵阳 550025)

\begin{abstract}
摘要 合成了一系列含有 $1,3,4$-噁二唑的杨梅素衍生物, 所有化合物经 ${ }^{1} \mathrm{H} N \mathrm{NM},{ }^{13} \mathrm{C} \mathrm{NMR}$ 以及 HRMS 表征. 生物活性 测试表明, 部分化合物对柑橘溃疡病菌 $(X a c)$ 、水稻白叶枯病菌 $(X o o)$ 以及烟草花叶病病毒 $(T M V)$ 具有较好的抑制作用. 其中化合物 4a、4b、4f、4j 对柑橘溃疡病菌的 $\mathrm{EC}_{50}$ 分别为 $18.5 、 40.7 、 26.9$ 和 $32.4 \mu \mathrm{g} / \mathrm{mL}$, 优于对照药叶枯唑 $(68.8 \mu \mathrm{g} / \mathrm{mL})$; 化合物 $\mathbf{4 f} 、 4 \mathbf{j}$ 对水稻白叶枯的 $\mathrm{EC}_{50}$ 分别为 45.9 和 $35.7 \mu \mathrm{g} / \mathrm{mL}$, 优于对照药叶枯唑 $(69.3 \mu \mathrm{g} / \mathrm{mL})$; 对 $T M V$ 治疗活性, 化 合物 $4 \mathbf{n}$ 的 $\mathrm{EC}_{50}$ 值为 $272.8 \mu \mathrm{g} / \mathrm{mL}$ ，优于对照药宁南霉素 $(428.8 \mu \mathrm{g} / \mathrm{mL})$; 对 $T M V$ 保护活性，化合物 $\mathbf{4 f}$ 的 $\mathrm{EC}_{50}$ 值为 235.6 $\mu \mathrm{g} / \mathrm{mL}$, 优于对照药宁南霉素 $(447.9 \mu \mathrm{g} / \mathrm{mL})$. 化合物 $\mathbf{4 j}$ 与南方水稻黑条矮缩病毒 P9-1 作用的微量热涌动实验表明, 该 化合物与 P9-1 之间具有较强的相互作用.
\end{abstract}

关键词 杨梅素; 噁二唑; 生物活性; 蛋白

\section{Synthesis and Biological of Novel Myricetin Derivatives Containing 1,3,4-Oxadiazoles}

\author{
Zhang, Cheng Jiang, Shichun Chen, Ying Guo, Tao Xia, Rongjiao \\ Tang, Xu Chen, Lijuan He, Ming Xue, Wei*
}

(State Key Laboratory Breeding Base of Green Pesticide and Agricultural Bioengineering, Key Laboratory of Green Pesticide and Agricultural Bioengineering, Ministry of Education, Center for Research and Development of Fine Chemicals, Guizhou University, Guiyang 550025)

\begin{abstract}
A series of novel myricetin derivatives containing 1,3,4-oxadiazole moiety were designed and synthesized. Bioassays indicated that some compounds showed potential antibacterial and antiviral activities. Among them, compounds $\mathbf{4 a}, \mathbf{4 b}, \mathbf{4 f}$ and $\mathbf{4 j}$ demonstrated appreciable inhibitory effect against Xanthomonas axonopodis pv.citri (Xac), with half-maximal effective concentration $\left(\mathrm{EC}_{50}\right)$ values of $18.5,40.7,26.9$ and $32.4 \mu \mathrm{g} / \mathrm{mL}$, which were significantly better than commercial agent bismerthiazol $(68.8 \mu \mathrm{g} / \mathrm{mL})$, compounds $\mathbf{4 f}$ and $\mathbf{4 j}$ also demonstrated appreciable inhibitory effect against Xanthomonas oryzae pv. Oryzae (Xoo) with $\mathrm{EC}_{50}$ values of 45.9 and $35.7 \mu \mathrm{g} / \mathrm{mL}$, which were better than commercial agent bismerthiazol (69.3 $\mu \mathrm{g} / \mathrm{mL}$ ). In addition, compounds $4 \mathrm{n}$ demonstrated significant curative activity against TMV with $\mathrm{EC}_{50}$ value of $272.8 \mu \mathrm{g} / \mathrm{mL}$, which was better than commercial agent ningnamycin $(428.8 \mu \mathrm{g} / \mathrm{mL})$, compounds $\mathbf{4 f}$ showed protecting activity against tobacco mosaic virus $(T M V)$ with $\mathrm{EC}_{50}$ value of $235.6 \mu \mathrm{g} / \mathrm{mL}$, which was better than commercial agent ningnamycin $(447.9 \mu \mathrm{g} / \mathrm{mL})$. Microscale thermophoresis (MST) indicated that compound $\mathbf{4 j}$ could bind with south rice black drawf virus P9-1.

Keywords myricetin; oxadiazole; biological activity; protein
\end{abstract}

植物病害会造成巨大的经济损失，例如烟草花叶病 毒 $(T M V)$, 能轻易感染烟草、番茄、黄瓜等经济农作物, 每年造成约 1 亿美元的损失 ${ }^{[1]}$; 南方水稻黑条矮缩病毒
$(S R B S D V)$ 是近年来新发现的斐济病毒, 能导致水稻、玉 米等作物出现发育迟缓、黑叶等症状 ${ }^{[2,3]}$; 水稻白叶枯病 菌 $(X o o)$ 在水稻的分㮐期侵染叶片而导致白叶枯病, 造

\footnotetext{
* Corresponding author. E-mail: wxue@gzu.edu.cn

Received September 28, 2018; revised November 2, 2018; published online December 17, 2018.

Project supported by the National Key Research and Development Program of China (No. 2017YFD0200506), and the National Natural Science Foundation of China (No. 21867003).

国家重点研发计划(No. 2017YFD0200506)、国家自然科学基金(No. 21867003)资助项目.
} 
成 $80 \%$ 的减产 $\left.{ }^{4}\right]$. 传统农药的使用, 虽然对植物病害起 到了一定的控制, 但由于其高毒、低活性以及农药残留 等问题, 已经渐渐被人们淘汰. 因此, 开发新型农药已 经是大势所趋.

天然产物又称次级代谢产物, 它的化学成分种类繁 多、结构新颖且具有独特的生物活性, 使之成为开发新 农药先导的热门 ${ }^{[5,6]}$. 黄酮类化合物是植物生长过程中 产生的一种次生代谢产物, 是一种重要的天然产物, 具 有广泛的生物活性和药理作用 ${ }^{[7]}$. 杨梅素是一种多羟基 黄酮类化合物, 具有抗病毒 ${ }^{[8 \sim 10]}$ 、抑菌 ${ }^{[11 \sim 13]}$ 、抗氧 化 ${ }^{[14,15]}$ 、抗癌细胞 ${ }^{[16,17]}$ 等作用, 我们课题组以杨梅素和 黄酮类化合物为先导体, 发现了一系列具有较好抑菌和
抗病毒活性的新颖的杨梅素和黄酮类衍生物 ${ }^{[10,13,16,18]}$.

1,3,4-噁二唑类化合物是一种重要的杂环化合物, 因其具有广泛的生物活性和药理作用等特点, 引起了广 泛的关注 ${ }^{[19]}$. 目前, 人们报道了 1,3,4-噁二唑类化合物, 具有抗菌 ${ }^{[20]}$ 、抗病毒 ${ }^{[21,22]}$ 、抗癌 ${ }^{[23,24]}$ 等生物活性. 同时 研究发现，对 1,3,4-噁二唑的结构进行修饰，会对它的 生物活性引起很大的变化 ${ }^{[25]}$.

鉴于此, 我们利用活性拼接原理将 2-氨基-1,3,4-噁 二唑基团引入杨梅素(Scheme 1), 设计合成了 16 个含啞 二坐基团的杨梅素新型化合物(合成路线见 Scheme 2). 通过浊度法和半叶枯斑法, 测试目标化合物的抑菌和抗 病毒活性，部分化合物的抑菌、抗病毒活性优于商品对

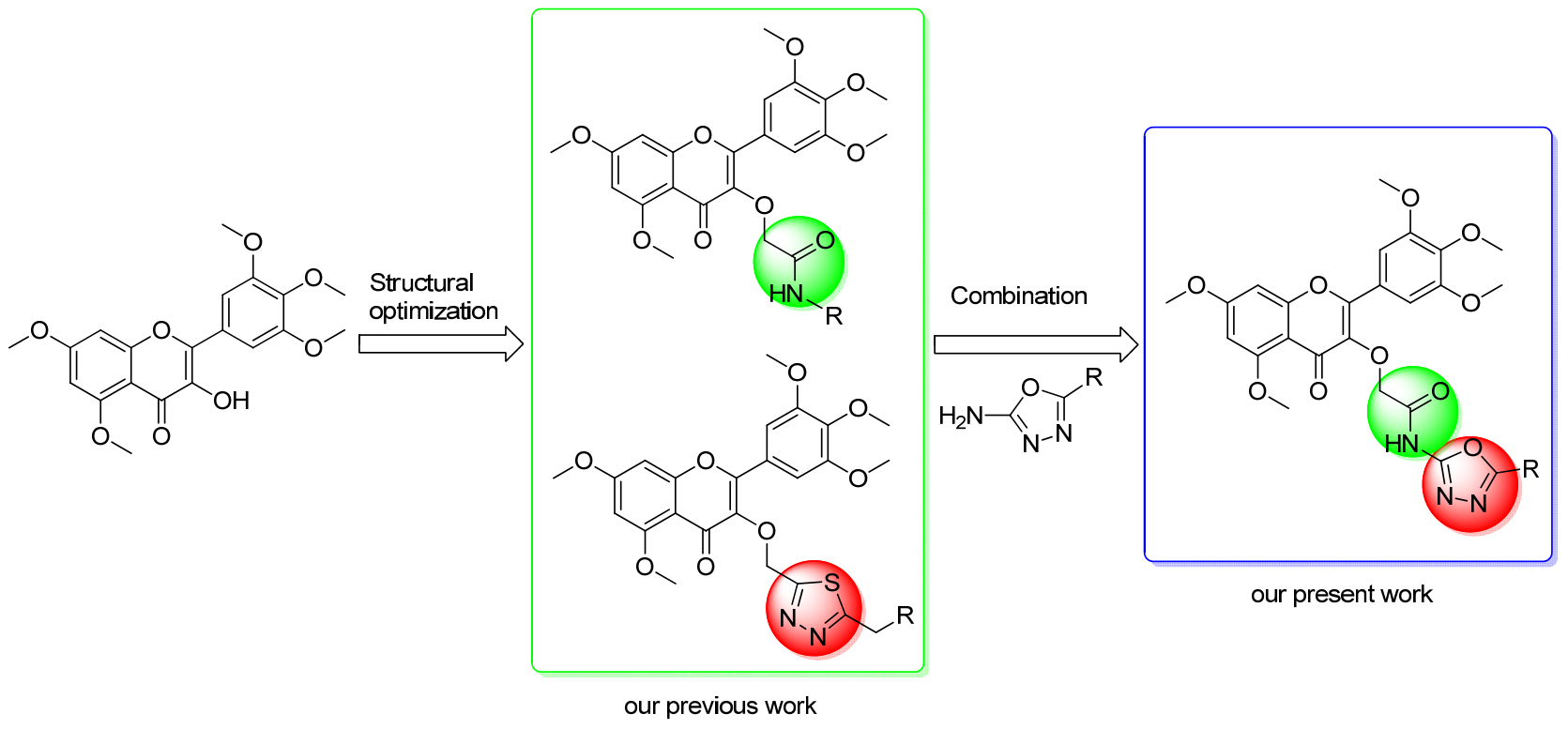

图式 1 目标分子的设计

Scheme 1 Design strategy for target molecules<smiles>[R]c1ccc(C([CH2+])=O)cc1</smiles><smiles>COc1cc(OC)c2c(=O)c(O)c(-c3cc(OC)c(OC)c(OC)c3)oc2c1</smiles><smiles>[R]c1ccc(-c2nnc(NC(=O)COc3c(-c4cc(OC)c(OC)c(OC)c4)oc4cc(OC)cc(OC)c4c3=O)o2)cc1</smiles>

a: $\mathrm{R}=\mathrm{H}, \mathbf{b}: \mathrm{R}=4-\mathrm{CH}_{3}, \mathbf{c}: \mathrm{R}=4-\mathrm{OCH}_{3}, \mathbf{d}: \mathrm{R}=4$-tert-butyl,

e: $\mathrm{R}=4-\mathrm{Cl}, \mathbf{f}: \mathrm{R}=2-\mathrm{OCH}_{3}, \mathbf{g :}: \mathrm{R}=2,4-\left(\mathrm{OCH}_{3}\right)_{2}$,

h: $\mathrm{R}=4$-isopropyl, i: $\mathrm{R}=3,4-\left(\mathrm{CH}_{3}\right)_{2}, \mathrm{j}: \mathrm{R}=3-\mathrm{CH}_{3}$,

k: $\mathrm{R}=3,4-\mathrm{Cl}_{2}$, I: $\mathrm{R}=4-\mathrm{Br}, \mathbf{m}: \mathrm{R}=2,4-\mathrm{Cl}_{2}$,

n: $\mathrm{R}=3,4-\left(\mathrm{OCH}_{3}\right)_{2}, \mathbf{o}: \mathrm{R}=3-\mathrm{Br}, \mathbf{p}: \mathrm{R}=2-\mathrm{Br}$

图式 2 目标化合物的合成路线

Scheme 2 Synthesis of target compounds 
照药剂宁南霉素和叶枯唑, 具有进一步研究的潜质.

\section{1 结果与讨论}

\section{1 中间体 2 的合成}

根据参考文献[26], 以苯为溶剂, 在回流条件下, 可以得到中间体 2 . 由于苯是致癌物质, 故放弃该方法; 通过笁选反应条件, 用 $N, N$-二甲基甲酰胺(DMF)为溶 剂, 再加入适量的二氯甲烷, 以碳酸钾为缚酸剂, 在冰 浴条件下过夜，得到较高收率的中间体 2 .

\section{2 目标化合物的抑菌活性测试}

目标化合物的抑菌活性测试结果见表 1. 实验表明, 所有化合物对供试的植物细菌都有一定的抑制作用. 其 中, 当浓度为 $100 \mu \mathrm{g} / \mathrm{mL}$, 化合物 4a、4b、4f 对柑橘溃 疡病菌 $(X a c)$ 的抑制作用均超过叶枯唑 $(63.3 \%)$; 化合物 4c、4f、4j 对水稻白叶枯病菌 $(X o o)$ 的抑制作用均超过叶 枯唑 $(56.0 \%)$.

以叶枯唑(Bismerthiazol)为对照药剂，测试 4a、4b、 $\mathbf{4 d} 、 \mathbf{4 f} 、 4 \mathbf{j}$ 的 $\mathrm{EC}_{50}$ 值，结果如表 2 所示，上述 5 个化合 物对柑橘溃疡病菌的 $\mathrm{EC}_{50}$ 值分别为 $18.5 、 40.7 、 57.0$ 、 $26.9 、 32.4 \mu \mathrm{g} / \mathrm{mL}$, 优于叶枯唑 $(68.8 \mu \mathrm{g} / \mathrm{mL})$; 同样, 以叶 枯唑(Bismerthiazol) 为对照药剂, 测试 $\mathbf{4 c} 、 \mathbf{4 f} 、 \mathbf{4 j}$ 的 $\mathrm{EC}_{50}$ 值, 结果如表 3 所示, 上述 3 个化合物对水稻白叶枯病 菌的 $\mathrm{EC}_{50}$ 值分别为 $66.3 、 45.9 、 35.7 \mu \mathrm{g} / \mathrm{mL}$, 优于叶枯 唑 $(69.3 \mu \mathrm{g} / \mathrm{mL})$.

抑菌活性测试表明，大部分化合物都具有一定的抑 菌活性. 构效关系分析表明，当取代基团为 $\mathrm{H}, 4-\mathrm{CH}_{3}$, $2-\mathrm{OCH}_{3}, 3-\mathrm{CH}_{3}$ 时, 目标化合物对 $X a c$ 表现出了很好的 抑制率, 如 $\mathbf{4 a}, \mathbf{4 b}, \mathbf{4 f}, \mathbf{4 j}$, 它们的 $\mathrm{EC}_{50}$ 值分别为 18.5 , $40.7,26.9,32.4 \mu \mathrm{g} / \mathrm{mL}$ ，优于对照药剂叶枯唑; 当取代基 团为 $4-\mathrm{OCH}_{3}, 2-\mathrm{OCH}_{3}, 3-\mathrm{CH}_{3}$ 时，目标化合物对 Xoo 表 现出了很好的抑制率, 如 $\mathbf{4 c}, \mathbf{4 f}, \mathbf{4 j}$, 它们的 $\mathrm{EC}_{50}$ 值分别 为 $66.3,45.9,35.7 \mu \mathrm{g} / \mathrm{mL}$, 优于对照药剂叶枯唑; 但是, 当取代基为 $2,4-\left(\mathrm{OCH}_{3}\right)_{2}(\mathbf{4 g}), 3,4-\left(\mathrm{CH}_{3}\right)_{2}$ (4i)，3,4$\left(\mathrm{OCH}_{3}\right)_{2}(\mathbf{4 n})$ 以及卤素原子时，目标化合物的抑菌活性

表 1 目标化合物 $\mathbf{4 a} \sim \mathbf{4 p}$ 的抑菌活性(抑制率/\% $)^{a}$

Table 1 Antibacterial activities (inhibition rate/\%) of target compound $\mathbf{4 a} \sim \mathbf{4 p}$

\begin{tabular}{|c|c|c|c|c|c|}
\hline \multirow{2}{*}{ Compound } & \multirow{2}{*}{$\mathrm{R}$} & \multicolumn{2}{|c|}{ Xanthomonas axonopodis pv. citri } & \multicolumn{2}{|c|}{ Xanthomonas oryzae pv. Oryzae } \\
\hline & & $100 \mu \mathrm{g} / \mathrm{mL}$ & $50 \mu \mathrm{g} / \mathrm{mL}$ & $100 \mu \mathrm{g} / \mathrm{mL}$ & $50 \mu \mathrm{g} / \mathrm{mL}$ \\
\hline $4 a$ & $\mathrm{H}$ & $94.6 \pm 3.3$ & $68.7 \pm 2.2$ & $29.7 \pm 1.8$ & $29.7 \pm 4.0$ \\
\hline $4 b$ & $4-\mathrm{CH}_{3}$ & $65.1 \pm 1.1$ & $50.0 \pm 0.7$ & $30.0 \pm 1.7$ & $29.9 \pm 3.8$ \\
\hline $4 c$ & $4-\mathrm{OCH}_{3}$ & $42.4 \pm 4.1$ & $22.2 \pm 1.8$ & $64.0 \pm 5.8$ & $41.5 \pm 6.8$ \\
\hline 4d & 4-tert-Butyl & $58.8 \pm 2.9$ & $46.2 \pm 3.3$ & $42.0 \pm 8.0$ & $30.8 \pm 3.2$ \\
\hline $4 e$ & $4-\mathrm{Cl}$ & $24.6 \pm 1.5$ & $17.3 \pm 0.5$ & $31.2 \pm 3.4$ & $22.4 \pm 2.6$ \\
\hline 4f & $2-\mathrm{OCH}_{3}$ & $86.1 \pm 1.5$ & $58.0 \pm 0.9$ & $72.4 \pm 3.0$ & $48.8 \pm 7.1$ \\
\hline $4 g$ & $2,4-\left(\mathrm{OCH}_{3}\right)_{2}$ & $9.9 \pm .3$ & $7.7 \pm 3.1$ & $39.2 \pm 2.5$ & $15.6 \pm 3.6$ \\
\hline $4 h$ & 4-Isopropyl & $19.2 \pm 3.2$ & $14.2 \pm 3.5$ & $49.2 \pm 1.2$ & $30.0 \pm 1.7$ \\
\hline $4 \mathbf{i}$ & $3,4-\left(\mathrm{CH}_{3}\right)_{2}$ & $28.5 \pm 2.0$ & $18.1 \pm 1.6$ & $19.8 \pm 4.3$ & $16.1 \pm 1.2$ \\
\hline $4 \mathbf{j}$ & $3-\mathrm{CH}_{3}$ & $61.6 \pm 1.5$ & $50.5 \pm 4.2$ & $94.2 \pm 1.4$ & $52.6 \pm 2.3$ \\
\hline $4 k$ & $3,4-\mathrm{Cl}_{2}$ & $31.1 \pm 1.9$ & $10.1 \pm 2.9$ & $40.5 \pm 4.6$ & $17.9 \pm 1.5$ \\
\hline 41 & $4-\mathrm{Br}$ & $9.7 \pm 2.4$ & $7.1 \pm 2.9$ & $47.9 \pm 3.5$ & $24.1 \pm 3.5$ \\
\hline $4 m$ & $2,4-\mathrm{Cl}_{2}$ & $26.1 \pm 2.2$ & $13.0 \pm 2.0$ & $44.5 \pm 3.4$ & $24.4 \pm 6.9$ \\
\hline $4 n$ & $3,4-\left(\mathrm{OCH}_{3}\right)_{2}$ & $35.6 \pm 6.8$ & $16.6 \pm 1.8$ & $41.3 \pm 2.8$ & $28.0 \pm 2.6$ \\
\hline 40 & $3-\mathrm{Br}$ & $19.4 \pm 0.9$ & $18.5 \pm 2.3$ & $31.1 \pm 1.8$ & $19.7 \pm 2.7$ \\
\hline $4 p$ & $2-\mathrm{Br}$ & $21.2 \pm 1.1$ & $14.3 \pm 2.3$ & $25.7 \pm 0.7$ & $13.9 \pm 1.0$ \\
\hline $\mathrm{MYR}^{b}$ & - & $42.1 \pm 2.3$ & $30.2 \pm 3.0$ & $47.1 \pm 2.3$ & $28.2 \pm 1.5$ \\
\hline Bismerthiazol $^{c}$ & - & $63.3 \pm 1.1$ & $41.5 \pm 1.6$ & $56.0 \pm 1.2$ & $33.8 \pm 2.0$ \\
\hline
\end{tabular}

${ }^{a}$ Average of three replicates. ${ }^{b}$ The lead compound of myricetin. ${ }^{c}$ The commercial bactericide (Bismerthizol) was used for comparison of antibacterial activity.

表 2 目标化合物 $4 a 、 4 b 、 4 d 、 4 f$ 和 $4 j$ 对柑橘溃疡病菌的抑制作用 ${ }^{a}$

Table 2 Inhibitory effects of compounds $\mathbf{4 a}, \mathbf{4 b}, \mathbf{4 d}, \mathbf{4} \mathbf{f}$ and $\mathbf{4 j}$ against Xanthomonas axonopodis pv. citri

\begin{tabular}{llccc}
\hline Compound & $\mathrm{R}$ & Toxic regression equation & $r$ & $\mathrm{EC}_{50} /\left(\mu \mathrm{g} \cdot \mathrm{mL}^{-1}\right)$ \\
\hline $\mathbf{4 a}$ & $\mathrm{H}$ & $y=1.1662 x+3.5310$ & 0.9623 & 18.5 \\
$\mathbf{4 b}$ & $4-\mathrm{CH}_{3}$ & $y=0.7203 x+3.8400$ & 0.9920 & 40.7 \\
$\mathbf{4 d}$ & $4-$ tert-Butyl & $y=0.7785 x+3.6328$ & 0.9938 & 57.0 \\
$\mathbf{4} \mathbf{f}$ & $2-\mathrm{OCH}_{3}$ & $y=1.6223 x+2.6802$ & 0.9804 & 26.9 \\
$\mathbf{4 j}$ & $3-\mathrm{CH}_{3}$ & $y=0.4471 x+4.3244$ & 0.9708 & 32.4 \\
Bismerthiazol $^{b}$ & - & $y=1.2051 x+2.7851$ & 0.9726 & 68.8 \\
\hline
\end{tabular}

${ }^{a}$ Average of three replicates. ${ }^{b}$ The commercial bactericide (Bismerthizol) was used for comparison of antibacterial activity. 
表 3 目标化合物 $4 \mathbf{c} 、 4 \mathbf{f}$ 和 $\mathbf{4 j}$ 对水稻白叶枯病菌的抑制作用 ${ }^{a}$

Table 3 Inhibitory effects of compounds $\mathbf{4 c}, \mathbf{4} \mathbf{f}$ and $\mathbf{4 j}$ against Xanthomonas oryzae pv. Oryzae

\begin{tabular}{ccccc}
\hline Compound & $\mathrm{R}$ & Toxic regression equation & $r$ & $\mathrm{EC}_{50} /\left(\mu \mathrm{g} \bullet \mathrm{mL}^{-1}\right)$ \\
\hline $\mathbf{4 c}$ & $4-\mathrm{OCH}_{3}$ & $y=1.6378 x+2.0161$ & 0.9846 & 66.3 \\
$\mathbf{4 f}$ & $2-\mathrm{OCH}_{3}$ & $y=1.6652 x+2.2318$ & 0.9851 & 45.9 \\
$\mathbf{4 j}$ & $3-\mathrm{CH}_{3}$ & $y=2.8364 x+0.5935$ & 0.9635 & 35.7 \\
Bismerthiazol $^{b}$ & - & $y=2.8364 x+0.5935$ & 0.9897 & 69.3 \\
\hline
\end{tabular}

${ }^{a}$ Average of three replicates. ${ }^{b}$ The commercial bactericide (Bismerthizol) was used for comparison of antibacterial activity.

比较差，即当取代基为 $\mathrm{H}$ 原子或是单取代的供电子基团 时，目标化合物的抑菌活性远远大于双取代的供电子基 才和卤素基团.

\section{3 目标化合物的抗 $T M V$ 活性}

目标化合物的抗 $T M V$ 活性测试结果如表 4 所示, 在 药剂浓度为 $500 \mu \mathrm{g} / \mathrm{mL}$ 时, 大部分化合物对 $T M V$ 病毒都 具有一定的抑制作用. 其中, 在治疗活性方面, 化合物 4a、 $4 \mathbf{n}$ 的抑制作用分别为 $57.1 \% 、 62.9 \%$, 优于对照药 宁南霉素(53.2\%), 在保护活性方面, 化合物 4d、 $4 \mathbf{f}$ 的抑 制作用分别为 $56.0 \% 、 65.3 \%$, 优于宁南霉素( $55.7 \%$ ).

以宁南霉素为对照药剂, 对化合物 $4 a 、 4 b 、 4 l$ 和 4n 进行抗 $T M V$ 的治疗活性 $\mathrm{EC}_{50}$ 测试, 结果见表 5, 它 们的 $\mathrm{EC}_{50}$ 值分别为 $362.6 、 385.0 、 502.5$ 和 $272.8 \mu \mathrm{g} / \mathrm{mL}$. 其中, 化合物 $4 \mathbf{a} 、 4 \mathbf{b}$ 和 $4 \mathbf{n}$ 的 $\mathrm{EC}_{50}$ 优于宁南霉素(428.8 $\mu \mathrm{g} / \mathrm{mL})$. 同样, 对化合物 $4 b 、 4 d 、 4 e 、 4 f$ 和 41 进行抗 $T M V$ 的保护活性 $\mathrm{EC}_{50}$ 测试, 结果见表 6 , 它们的 $\mathrm{EC}_{50}$ 值分别为 $626.2 、 404.0 、 406.0 、 235.6$ 和 $562.0 \mu \mathrm{g} / \mathrm{mL}$. 其 中, 化合物 $4 \mathbf{f}$ 的 $\mathrm{EC}_{50}$ 值远远优于宁南需素(447.9 $\mu \mathrm{g} / \mathrm{mL})$.
表 4 目标化合物 $\mathbf{4 a} \sim \mathbf{4 p}$ 的抗 TMV 活性 ${ }^{a}$

Table 4 Antiviral activities of target compound $\mathbf{4 a} \sim \mathbf{4 p}$ against $T M V$

\begin{tabular}{|c|c|c|c|}
\hline Compound & $\mathrm{R}$ & Curative activity $/ \%$ & $\begin{array}{l}\text { Protection } \\
\text { activity } / \%\end{array}$ \\
\hline $4 a$ & $\mathrm{H}$ & 57.1 & 40.9 \\
\hline $4 b$ & $4-\mathrm{CH}_{3}$ & 51.3 & 48.0 \\
\hline $4 c$ & $4-\mathrm{OCH}_{3}$ & 40.9 & 23.3 \\
\hline $4 d$ & 4-tert-butyl & 26.8 & 56.0 \\
\hline $4 e$ & $4-\mathrm{Cl}$ & 32.0 & 51.7 \\
\hline $4 f$ & $2-\mathrm{OCH}_{3}$ & 37.0 & 65.3 \\
\hline $4 g$ & $2,4-\left(\mathrm{OCH}_{3}\right)_{2}$ & 44.5 & 18.2 \\
\hline $4 h$ & 4-Isopropyl & 31.6 & 35.8 \\
\hline $4 i$ & $3,4-\left(\mathrm{CH}_{3}\right)_{2}$ & 28.2 & 27.8 \\
\hline $4 j$ & $3-\mathrm{CH}_{3}$ & 42.8 & 27.5 \\
\hline $4 k$ & $3,4-\mathrm{Cl}_{2}$ & 35.0 & 30.0 \\
\hline 41 & $4-\mathrm{Br}$ & 50.7 & 48.7 \\
\hline $4 m$ & $2,4-\mathrm{Cl}_{2}$ & 39.8 & 17.5 \\
\hline $4 n$ & $3,4-\left(\mathrm{OCH}_{3}\right)_{2}$ & 62.9 & 34.0 \\
\hline 40 & $3-\mathrm{Br}$ & 35.4 & 16.8 \\
\hline $4 p$ & $2-\mathrm{Br}$ & 37.8 & 19.2 \\
\hline $\mathrm{MYR}^{b}$ & & 35.7 & 41.5 \\
\hline Ningnamycin $^{c}$ & & 53.2 & 55.7 \\
\hline
\end{tabular}

表 5 目标化合物 $4 \mathrm{a} 、 4 \mathrm{~b} 、 4 \mathrm{l}$ 和 $4 \mathrm{n}$ 对 $T M V$ 的治疗活性 ${ }^{a}$

Table 5 Curative activity of compounds $4 a, 4 b, 4 l$ and $4 \mathrm{n}$ against TMV

\begin{tabular}{|c|c|c|c|c|}
\hline Compound & $\mathrm{R}$ & Toxic regression equation & $r$ & $\mathrm{EC}_{50} /\left(\mu \mathrm{g} \cdot \mathrm{mL}^{-1}\right)$ \\
\hline $4 \mathbf{a}$ & $\mathrm{H}$ & $y=1.0698 x+2.2618$ & 0.9893 & 362.6 \\
\hline $4 b$ & $4-\mathrm{CH}_{3}$ & $y=1.9125 x-0.0551$ & 0.9710 & 385.0 \\
\hline 41 & $4-\mathrm{Br}$ & $y=1.2688 x+1.5728$ & 0.9922 & 502.5 \\
\hline $4 n$ & $3,4-\left(\mathrm{OCH}_{3}\right)_{2}$ & $y=1.1024 x+2.3146$ & 0.9769 & 272.8 \\
\hline Ningnamycin ${ }^{b}$ & - & $y=0.7650 x+2.9863$ & 0.9830 & 428.8 \\
\hline
\end{tabular}

${ }^{a}$ Average of three replicates. ${ }^{b}$ The commercial antivirotic (Ningnamycin) was used for comparison of antiviral activity.

表 6 目标化合物 $4 \mathrm{~b} 、 4 \mathrm{~d} 、 4 \mathrm{e} 、 4 \mathrm{f}$ 和 $4 \mathrm{l}$ 对 $T M V$ 的保护活性 ${ }^{a}$

Table 6 Protective activity of compounds $4 \mathrm{~b}, \mathbf{4 d}, \mathbf{4 e}, \mathbf{4 f}$ and $\mathbf{4 l}$ against TMV

\begin{tabular}{|c|c|c|c|c|}
\hline Compound & $\mathrm{R}$ & Toxic regression equation & $r$ & $\mathrm{EC}_{50} /\left(\mu \mathrm{g} \cdot \mathrm{mL}^{-1}\right)$ \\
\hline $4 b$ & $4-\mathrm{CH}_{3}$ & $y=0.3375 x+4.0561$ & 0.9600 & 626.2 \\
\hline 4d & 4-tert-Butyl & $y=1.2188 x+1.8227$ & 0.9837 & 404.0 \\
\hline $4 e$ & $4-\mathrm{Cl}$ & $y=0.8601 x+2.7564$ & 0.9832 & 406.0 \\
\hline $4 f$ & $2-\mathrm{OCH}_{3}$ & $y=1.0608 x+2.4835$ & 0.9786 & 235.6 \\
\hline 41 & $4-\mathrm{Br}$ & $y=1.3146 x+1.3951$ & 0.9973 & 562.0 \\
\hline Ningnamycin ${ }^{b}$ & - & $y=1.5482 x+0.8954$ & 0.9819 & 447.9 \\
\hline
\end{tabular}

${ }^{a}$ Average of three replicates. ${ }^{b}$ The commercial antivirotic (Ningnamycin) was used for comparison of antiviral activity. 


\section{4 目标化合物与 P9-1 微量热涌动实验分析}

以目标化合物 $\mathbf{4 j}$ 为代表, 研究含酰胺噁二唑的杨 梅素衍生物的初步抗水稻病毒机制, 通过微量热涌动实 验(MST) NT.115 分析软件得到 $\mathbf{4 j} 、$ MYR 与 P9-1 发生相 互作用的荧光光谱(图 1)和解离常数, 通过分析 $4 \mathbf{j}$ 的浓 度与相对荧光 $F_{\text {norm }}$ 的关系, 发现化合物 $4 \mathbf{j}$ 与 P9-1 的解 离常数 $K_{\mathrm{d}}=(0.049 \pm 0.017) \mu \mathrm{mol} / \mathrm{L}$, 远远优于先导化合 物杨梅素的解离常数 $K_{\mathrm{d}}=(7.789 \pm 5.678) \mu \mathrm{mol} / \mathrm{L}$ (图 2), 说明了它们之间有很好的结合作用.
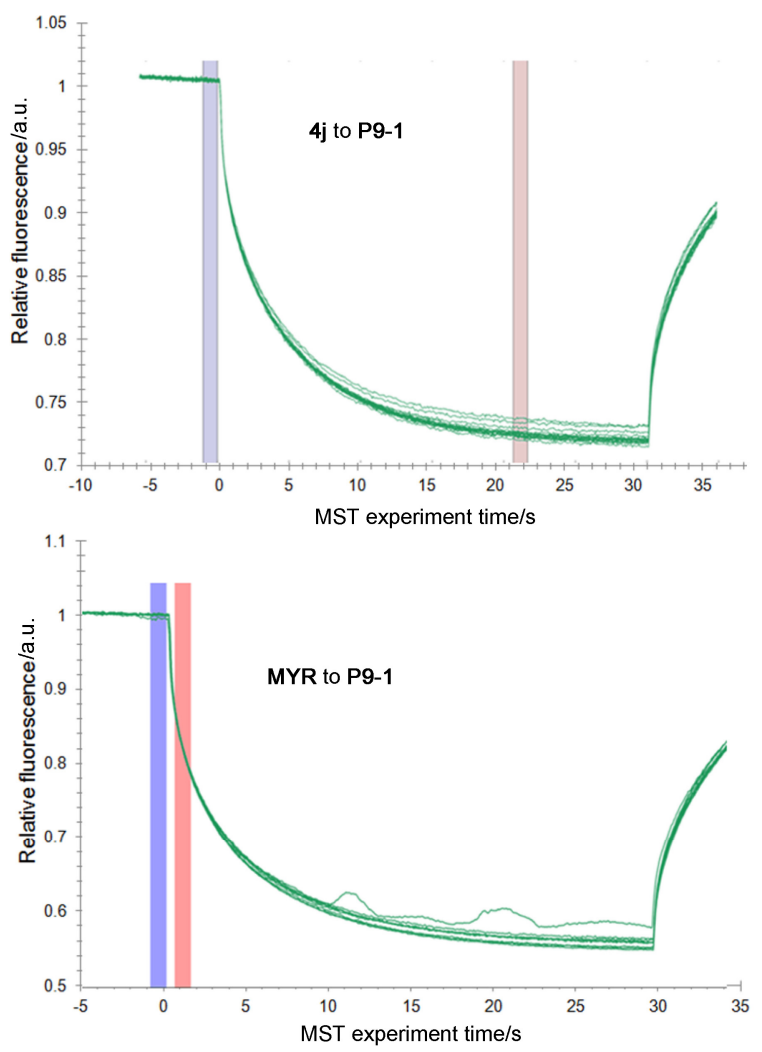

图 $14 \mathbf{j} 、 M R Y$ 与 P9-1 相互作用的荧光图谱

Figure 1 Fluorescence spectra of interaction between $\mathbf{4 j}$, MYR and P9-1

\section{2 结论}

本文以杨梅苷、氨基脲盐酸盐和取代苯甲醛为原料, 合成了 16 个含噁二唑的杨梅素衍生物. 生物活性测试 证明，大部分化合物对 $X o O$ 、 $X a c$ 以及 $T M V$ 都具有一定 的抑制作用. 其中化合物 $\mathbf{4 a}$ 对 $X a c$ 的抑制作用优于对照 药叶枯唑; 化合物 $\mathbf{4 j}$ 对 Xoo 的抑制作用优于对照药叶枯 唑; 化合物 4n 对 $T M V$ 治疗活性优于对照药宁南霉素; 化合物 4f 对 $T M V$ 保护活性优于对照药宁南霉素. 化合 物 4j 与南方水稻黑条矮缩病毒 P9-1 作用的微量热涌动 实验表明，该化合物与 P9-1 蛋白的结合力优于没有进 行分子修饰的杨梅素, 为研究杨梅素衍生物的抑菌抗病
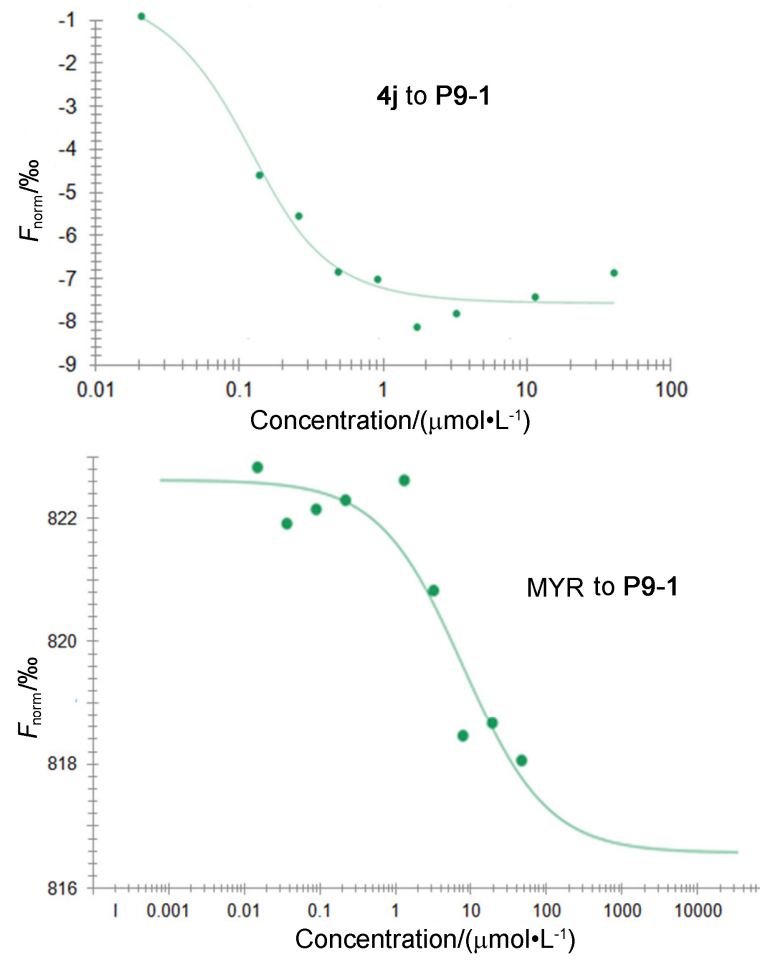

图 $24 j 、 M Y R$ 与 P9-1 的热力学曲线

Figure 2 Thermodynamic curves of $\mathbf{4 j}$, MYR and P9-1

毒的作用机制奠定了一定基础.

\section{3 实验部分}

\section{1 仪器与试剂}

${ }^{1} \mathrm{H}$ NMR、 ${ }^{13} \mathrm{C}$ NMR 由 Bruker Ascend-400 spectrometer, JEOL ECX-500 型核磁共振仪测定[TMS 为内 标, $\mathrm{CDCl}_{3}$ 或二甲基亚砜(DMSO)为溶剂]; 高分辨质谱 为美国 Thermofisher 公司 Q Exactive 型液质联用仪; 熔 点仪采用上海仪电物理光学仪器有限公司的 X-4B 熔点 仪. 氨基嫝盐酸盐、碘单质、取代苯甲醛等购自上海泰 坦科技股份有限公司，杨梅苷购自上海诺特生物科技有 限公司，其他试剂均为市售分析纯.

\section{2 实验方法}

3.2.1 中间体 $\mathbf{1 a} \sim \mathbf{1 p}$ 的合成方法

合成方法参照文献[27], 理化性质及谱图见辅助材 料.

\subsection{2中间体 $\mathbf{2 a} \sim \mathbf{2 p}$ 的合成方法}

向 $100 \mathrm{~mL}$ 圆底烧瓶中加入 $1(2.48 \mathrm{mmol})$ 、碳酸钾

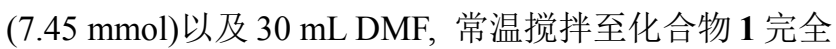
溶解后，加入 15 20 mL 二氯甲烷，常温搅拌 $0.5 \sim 1 \mathrm{~h}$, 冰浴条件下缓慢滴加氯乙酰氯 $(7.45 \mathrm{mmol})$, 反应过夜. 将二氯甲烷旋干后，将反应物倒入冰水中，得到中间体 2, 无需提纯, 直接进行下一步反应. 理化性质及谱图见 
辅助材料.

3.2.3中间体 3 的合成

中间体 3 的合成参照文献[16]的方法合成.

\subsection{4 目标化合物的合成}

向 $50 \mathrm{~mL}$ 圆底烧瓶中加入中间体 $3(1.03 \mathrm{mmol}) 、$ 碳 酸钾 $(3.09 \mathrm{mmol})$ 和 $\mathrm{DMF} 20 \mathrm{~mL}, 85{ }^{\circ} \mathrm{C}$ 下搅拌 $1 \mathrm{~h}$ 后, 缓 慢滴加 $10 \mathrm{~mL}$ 含有中间体 $2(1.04 \mathrm{mmol})$ 的 DMF, $105{ }^{\circ} \mathrm{C}$ 下搅拌 5 7 h, 将反应物倒入 $200 \mathrm{~mL}$ 水中, 在搅拌下缓 慢滴加 $6 \mathrm{~mol} / \mathrm{L}$ 的盐酸调节 $\mathrm{pH}$ 至酸性, 有大量固体析 出, 抽滤, 用二次水冲洗 2 3 次, DMF 和甲醇重结晶, 得到目标化合 4 .

2-[5,7-二甲氧基-4-酮-2-(3,4,5-三甲氧基苯基)- $4 H$ 色烯-3-基]氧基- $N$-(5-苯基-1,3,4-噁二唑-2-基) 乙酰胺 (4a): 灰色固体, 产率 47.1\%. m.p. $247.3 \sim 248.5{ }^{\circ} \mathrm{C} ;{ }^{1} \mathrm{H}$ NMR (500 MHz, $\left.\mathrm{CDCl}_{3}\right) \delta: 12.54(\mathrm{~s}, 1 \mathrm{H}), 8.09$ (dd, $J=$ 7.9, $1.6 \mathrm{~Hz}, 2 \mathrm{H}), 7.52 \sim 7.46(\mathrm{~m}, 3 \mathrm{H}), 7.26$ (s, 2H ), 6.57 $(\mathrm{d}, J=2.2 \mathrm{~Hz}, 1 \mathrm{H}), 6.43(\mathrm{~d}, J=2.2 \mathrm{~Hz}, 1 \mathrm{H}), 4.41(\mathrm{~s}, 2 \mathrm{H})$, $4.00(\mathrm{~s}, 3 \mathrm{H}), 3.96 \sim 3.96(\mathrm{~m}, 3 \mathrm{H}), 3.95(\mathrm{~s}, 6 \mathrm{H}), 3.94(\mathrm{~s}$, $3 \mathrm{H}) ;{ }^{13} \mathrm{C} \mathrm{NMR}\left(126 \mathrm{MHz}, \mathrm{CDCl}_{3}\right) \delta: 174.81,166.76$, $165.18,161.62,161.23,159.18,157.00,154.54,153.66$, $141.21,141.15,131.45,128.97,126.83,124.54,123.94$, 108.58, 106.01, 96.63, 92.85, 73.50, 61.18, 56.68, 56.62, 56.08; HRMS calcd for $\mathrm{C}_{30} \mathrm{H}_{28} \mathrm{~N}_{3} \mathrm{O}_{10}[\mathrm{M}+\mathrm{H}]^{+} 590.1769$, found 590.1759 .

2-[5,7-二甲氧基-4-酮-2-(3,4,5-三甲氧基苯基)- $4 H$ 色烯-3-基]氧基- $N$-[5-(4-甲基苯基)-1,3,4-噁二唑-2-基]乙 酰胺(4b): 灰色固体, 产率 40.4\%. m.p. 243.9 245.4 ${ }^{\circ} \mathrm{C}$; ${ }^{1} \mathrm{H}$ NMR (500 MHz, $\left.\mathrm{CDCl}_{3}\right) \delta: 12.47$ (s, 1H), 7.98 (d, $J=$ $8.2 \mathrm{~Hz}, 2 \mathrm{H}), 7.29$ (d, J=8.0 Hz, 2H), 7.26 (s, 2H), 6.57 (d, $J=2.2 \mathrm{~Hz}, 1 \mathrm{H}), 6.43(\mathrm{~d}, J=2.2 \mathrm{~Hz}, 1 \mathrm{H}), 4.41(\mathrm{~s}, 2 \mathrm{H}), 4.01$ $(\mathrm{s}, 3 \mathrm{H}), 3.96(\mathrm{~s}, 3 \mathrm{H}), 3.95(\mathrm{~s}, 6 \mathrm{H}), 3.94(\mathrm{~s}, 3 \mathrm{H}), 2.42(\mathrm{~s}$, $3 \mathrm{H}) ;{ }^{13} \mathrm{C} \mathrm{NMR}\left(101 \mathrm{MHz}, \mathrm{CDCl}_{3}\right) \delta: 174.74,166.70$, $165.09,161.70,161.11,159.09,156.68,154.44,153.56$, $141.88,141.13,140.98,129.61,126.71,124.48,121.04$, 108.48, 105.86, 96.55, 92.76, 73.41, 61.11, 56.61, 56.52, 56.03, 21.63; HRMS calcd for $\mathrm{C}_{31} \mathrm{H}_{30} \mathrm{~N}_{3} \mathrm{O}_{10}[\mathrm{M}+\mathrm{H}]^{+}$ 604.1925 , found 604.1913 .

2-[5,7-二甲氧基-4-酮-2-(3,4,5-三甲氧基苯基)- $4 H$ 色烯-3-基]氧基- $N$-[5-(4-甲氧基)苯基)-1,3,4-啞二唑-2基]乙酰胺(4c): 灰色固体，产率 39.2\%. m.p. 252.6 $253.9{ }^{\circ} \mathrm{C} ;{ }^{1} \mathrm{H}$ NMR (400 MHz, $\left.\mathrm{CDCl}_{3}\right) \delta$ : 12.40 (s, 1H), $8.03(\mathrm{~d}, J=8.5 \mathrm{~Hz}, 2 \mathrm{H}), 7.26(\mathrm{~s}, 2 \mathrm{H}), 6.99$ (d, $J=8.5 \mathrm{~Hz}$, 2H), 6.57 (s, 1H), $6.43(\mathrm{~s}, 1 \mathrm{H}), 4.41(\mathrm{~s}, 2 \mathrm{H}), 4.01(\mathrm{~s}, 3 \mathrm{H})$, $3.95(\mathrm{t}, J=4.1 \mathrm{~Hz}, 12 \mathrm{H}), 3.88(\mathrm{~s}, 3 \mathrm{H}) ;{ }^{13} \mathrm{C}$ NMR $(101$ $\left.\mathrm{MHz}, \mathrm{CDCl}_{3}\right) \delta: 174.52,166.52,164.90,161.94,161.41$,
$161.00,158.95,156.27,154.24,153.42,140.98,140.87$, $128.37,124.34,116.28,114.18,108.39,105.75,96.39$, 92.60, 73.23, 60.95, 56.47, 56.38, 55.84, 55.29; HRMS calcd for $\mathrm{C}_{31} \mathrm{H}_{30} \mathrm{~N}_{3} \mathrm{O}_{11}[\mathrm{M}+\mathrm{H}]^{+}$620.1874, found 620.1862 .

2-[5,7-二甲氧基-4-酮-2-(3,4,5-三甲氧基苯基)-4H色烯-3-基]氧基- $N$-[5-(4-叔丁基苯基)-1,3,4-噁二唑-2-基] 乙酰胺(4d): 灰色固体，产率 32.2\%. m.p. 241.3 $242.2{ }^{\circ} \mathrm{C} ;{ }^{1} \mathrm{H}$ NMR (500 MHz, $\left.\mathrm{CDCl}_{3}\right) \delta: 12.43$ (s, 1H), $8.02(\mathrm{~d}, J=8.5 \mathrm{~Hz}, 2 \mathrm{H}), 7.51$ (d, $J=8.5 \mathrm{~Hz}, 2 \mathrm{H}), 7.26$ (s, 2H), $6.57(\mathrm{~d}, J=2.2 \mathrm{~Hz}, 1 \mathrm{H}), 6.43(\mathrm{~d}, J=2.2 \mathrm{~Hz}, 1 \mathrm{H}), 4.42$ $(\mathrm{s}, 2 \mathrm{H}), 4.01(\mathrm{~s}, 3 \mathrm{H}), 3.96(\mathrm{~s}, 3 \mathrm{H}), 3.95(\mathrm{~s}, 6 \mathrm{H}), 3.94(\mathrm{~s}$, $3 \mathrm{H}), 1.35(\mathrm{~s}, 9 \mathrm{H}) ;{ }^{13} \mathrm{C} \mathrm{NMR}\left(126 \mathrm{MHz}, \mathrm{CDCl}_{3}\right) \delta: 174.75$, $166.73,165.14,161.76,161.23,159.18,156.74,154.99$, $154.46,153.65,141.19,141.12,126.66,125.93,124.57$, 121.12, 108.62, 106.00, 96.62, 92.84, 73.45, 61.17, 56.70, 56.61, 56.07, 35.12, 31.22; HRMS calcd for $\mathrm{C}_{34} \mathrm{H}_{36} \mathrm{~N}_{3} \mathrm{O}_{10}$ $[\mathrm{M}+\mathrm{H}]^{+}$646.2395, found 646.2383 .

2-[5,7-二甲氧基-4-酮-2-(3,4,5-三甲氧基苯基)-4H色烯-3-基]氧基- $N$-[5-(4-氯苯基)-1,3,4-噁二唑-2-基]乙酰 胺(4e): 灰色固体, 产率 35.8\%. m.p. 238.2 239.5 ${ }^{\circ} \mathrm{C}$; ${ }^{1} \mathrm{H}$ NMR (400 MHz, $\mathrm{CDCl}_{3}$ ) $\delta: 12.73(\mathrm{~s}, 1 \mathrm{H}), 8.02$ (t, $J=$ $10.0 \mathrm{~Hz}, 2 \mathrm{H}), 7.47$ (d, J=8.5 Hz, 2H), 7.26 (s, 2H), 6.58 $(\mathrm{d}, J=1.6 \mathrm{~Hz}, 1 \mathrm{H}), 6.43$ (s, 1H), $4.41(\mathrm{~s}, 2 \mathrm{H}), 4.01(\mathrm{~s}, 3 \mathrm{H})$, 3.97 (s, 3H), $3.96(\mathrm{~s}, 6 \mathrm{H}), 3.95(\mathrm{~s}, 3 \mathrm{H}) ;{ }^{13} \mathrm{C}$ NMR (101 $\left.\mathrm{MHz}, \mathrm{CDCl}_{3}\right) \delta: 174.81,166.73,165.13,161.09,160.64$, $159.10,157.05,154.52,153.57,141.15,140.99,137.60$, $129.30,128.01,124.42,122.31,108.42,105.84,96.59$, 92.77, 73.45, 61.13, 56.64, 56.52, 56.05; HRMS calcd for $\mathrm{C}_{30} \mathrm{H}_{27} \mathrm{ClN}_{3} \mathrm{O}_{10}[\mathrm{M}+\mathrm{H}]^{+}$624.1379, found 624.1369.

2-[5,7-二甲氧基-4-酮-2-(3,4,5-三甲氧基苯基)-4H色烯-3-基]氧基- $N$-[5-(2-甲氧基苯基)-1,3,4-噁二唑-2-基] 乙酰胺 (4f): 灰色固体, 产率 45.3\%. m.p. 222.3 $223.1{ }^{\circ} \mathrm{C}$; ${ }^{1} \mathrm{H}$ NMR (500 MHz, $\left.\mathrm{CDCl}_{3}\right) \delta: 12.28$ (s, 1H), $7.93(\mathrm{~d}, J=7.7 \mathrm{~Hz}, 1 \mathrm{H}), 7.48(\mathrm{t}, J=7.3 \mathrm{~Hz}, 1 \mathrm{H}), 7.25$ (s, $2 \mathrm{H}), 7.08 \sim 7.01(\mathrm{~m}, 2 \mathrm{H}), 6.56(\mathrm{~d}, J=1.8 \mathrm{~Hz}, 1 \mathrm{H}), 6.42(\mathrm{~d}$, $J=1.7 \mathrm{~Hz}, 1 \mathrm{H}), 4.42$ (s, 2H), 3.99 (s, 3H), 3.98 (s, 3H), 3.96 (s, 3H), 3.95 (s, 6H), $3.94(\mathrm{~s}, 3 \mathrm{H}) ;{ }^{13} \mathrm{C}$ NMR $(126$ $\left.\mathrm{MHz}, \mathrm{CDCl}_{3}\right) \delta: 174.80,166.92,165.27,161.43,160.60$, $159.35,158.22,156.97,154.55,153.83,141.30,141.27$, $133.00,130.80,124.81,120.89,113.45,112.16,108.86$, 106.18, 96.77, 93.01, 73.55, 61.36, 56.84, 56.80, 56.32, 56.25; HRMS calcd for $\mathrm{C}_{31} \mathrm{H}_{30} \mathrm{~N}_{3} \mathrm{O}_{11}[\mathrm{M}+\mathrm{H}]^{+}$620.1874, found 620.1868 .

2-[5,7-二甲氧基-4-酮-2-(3,4,5-三甲氧基苯基)- $4 H$ - 
色烯-3-基]氧基- $N$-[5-(2,4-二甲氧基苯基)-1,3,4-噁二唑2-基]乙酰胺(4g)：黄色固体，产率 43.6\%. m.p. 198.1 $199.5{ }^{\circ} \mathrm{C}$; ${ }^{1} \mathrm{H}$ NMR (500 MHz, DMSO- $\left.d_{6}\right) \delta$ : 11.95 (s, $1 \mathrm{H}), 7.71(\mathrm{~d}, J=8.6 \mathrm{~Hz}, 1 \mathrm{H}), 7.51(\mathrm{~s}, 2 \mathrm{H}), 6.92$ (d, $J=2.2$ $\mathrm{Hz}, 1 \mathrm{H}), 6.78$ (d, $J=2.3 \mathrm{~Hz}, 2 \mathrm{H}), 6.74(\mathrm{dd}, J=8.7,2.3 \mathrm{~Hz}$, $1 \mathrm{H}), 6.55$ (d, $J=2.2 \mathrm{~Hz}, 1 \mathrm{H}), 4.86(\mathrm{~s}, 2 \mathrm{H}), 3.94$ (s, 3H), 3.92 (s, 6H), 3.90 (s, 3H), 3.89 (s, 3H), 3.88 (s, 3H), 3.78 $(\mathrm{s}, 3 \mathrm{H}) ;{ }^{13} \mathrm{C}$ NMR (126 MHz, DMSO- $\left.d_{6}\right) \quad \delta: 172.82$, $167.44,164.59,163.91,160.85,160.33,159.35,158.71$, $156.94,153.29,152.00,140.19,139.86,131.58,125.66$, 108.67, 106.69, 106.57, 105.51, 99.64, 96.67, 93.72, 70.69, $60.71,56.71,56.67,56.54,56.18$; HRMS calcd for $\mathrm{C}_{32} \mathrm{H}_{32} \mathrm{~N}_{3} \mathrm{O}_{12}[\mathrm{M}+\mathrm{H}]^{+}$650.1980, found 650.1976.

2-[5,7-二甲氧基-4-酮-2-(3,4,5-三甲氧基苯基)- $4 H$ 色烯-3-基]氧基- $N$-[5-(4-异丙基苯基)-1,3,4-啞二唑-2-基] 乙酰胺 (4h): 灰色固体, 产率 43.1\%. m.p. 213.9 $214.3{ }^{\circ} \mathrm{C} ;{ }^{1} \mathrm{H}$ NMR (400 MHz, $\left.\mathrm{CDCl}_{3}\right) \delta$ : 12.54 (s, 1H), 8.01 (d, $J=8.2 \mathrm{~Hz}, 2 \mathrm{H}), 7.35$ (d, $J=8.3 \mathrm{~Hz}, 2 \mathrm{H}), 7.26$ (s, 2H), 6.57 (d, $J=2.1 \mathrm{~Hz}, 1 \mathrm{H}), 6.43$ (d, $J=2.1 \mathrm{~Hz}, 1 \mathrm{H}), 4.41$ $(\mathrm{s}, 2 \mathrm{H}), 4.01(\mathrm{~s}, 3 \mathrm{H}), 3.96(\mathrm{~s}, 3 \mathrm{H}), 3.95(\mathrm{~s}, 6 \mathrm{H}), 3.94(\mathrm{~s}$, $3 \mathrm{H}), 2.97(\mathrm{dt}, J=13.8,7.0 \mathrm{~Hz}, 1 \mathrm{H}), 1.29$ (d, $J=6.9 \mathrm{~Hz}$, $6 \mathrm{H}) ;{ }^{13} \mathrm{C} \mathrm{NMR}\left(101 \mathrm{MHz}, \mathrm{CDCl}_{3}\right) \delta: 174.73,166.69$, $165.08,161.69$, 161.11, 159.09, 156.68, 154.43, 153.56, $152.69,141.12$, 140.97, 127.02, 126.85, 124.48, 121.36, 108.48, 105.85, 96.55, 92.76, 73.40, 61.11, 56.62, 56.52, 56.03, 34.23, 23.74; HRMS calcd for $\mathrm{C}_{33} \mathrm{H}_{34} \mathrm{~N}_{3} \mathrm{O}_{10}[\mathrm{M}+$ $\mathrm{H}]^{+}$632.2238, found 632.2229 .

2-[5,7-二甲氧基-4-酮-2-(3,4,5-三甲氧基苯基)- $4 H$ 色烯-3-基]氧基- $N$-[5-(3,4-二甲基苯基)-1,3,4-噁二唑-2基]乙酰胺(4i): 灰色固体, 产率 37.4\%. m.p. 203.4 $213.8{ }^{\circ} \mathrm{C}$; ${ }^{1} \mathrm{H}$ NMR (500 MHz, DMSO- $d_{6}$ ) $\delta$ : 12.09 (s, 1H), $7.72(\mathrm{~s}, 1 \mathrm{H}), 7.67(\mathrm{~d}, J=7.8 \mathrm{~Hz}, 1 \mathrm{H}), 7.51(\mathrm{~s}, 2 \mathrm{H})$, 7.39 (d, $J=7.9 \mathrm{~Hz}, 1 \mathrm{H}), 6.94$ (d, $J=2.1 \mathrm{~Hz}, 1 \mathrm{H}), 6.57$ (d, $J=2.1 \mathrm{~Hz}, 1 \mathrm{H}), 4.87$ (s, 2H), 3.95 (s, 3H), 3.92 (s, 6H), 3.90 (s, 3H), 3.77 (s, 3H), 2.34 (d, J=4.9 Hz, 6H); ${ }^{13} \mathrm{C}$ NMR (101 MHz, DMSO- $\left.d_{6}\right) \delta: 172.78,167.41,164.53$, $161.45,160.77,158.66,157.22,153.21,151.93,141.19$, $140.06,139.83,138.11,130.91,127.18,125.61,124.00$, $121.31,108.58,106.45,96.62,93.65,70.70,60.64,56.65$, 56.60, 56.58, 19.99, 19.79; HRMS calcd for $\mathrm{C}_{32} \mathrm{H}_{32} \mathrm{~N}_{3} \mathrm{O}_{10}$ $[\mathrm{M}+\mathrm{H}]^{+}$618.2082, found 618.2078 .

2-[5,7-二甲氧基-4-酮-2-(3,4,5-三甲氧基苯基)- $4 H$ 色烯-3-基]氧基- $N$-[5-(3-甲基苯基)-1,3,4-噁二唑-2-基]乙 酰胺(4j): 灰色固体, 产率 45.3\%. m.p. 196.6 197.7 ${ }^{\circ} \mathrm{C}$; ${ }^{1} \mathrm{H}$ NMR (400 MHz, DMSO- $\left.d_{6}\right) \delta: 12.12(\mathrm{~s}, 1 \mathrm{H}), 7.72$ (d,
$J=11.8 \mathrm{~Hz}, 2 \mathrm{H}), 7.51 \sim 7.45(\mathrm{~m}, 3 \mathrm{H}), 7.43(\mathrm{~d}, J=7.6 \mathrm{~Hz}$, $1 \mathrm{H}), 6.90(\mathrm{~d}, J=2.2 \mathrm{~Hz}, 1 \mathrm{H}), 6.53(\mathrm{~d}, J=2.2 \mathrm{~Hz}, 1 \mathrm{H}), 4.85$ $(\mathrm{s}, 2 \mathrm{H}), 3.91(\mathrm{~s}, 3 \mathrm{H}), 3.88(\mathrm{~s}, 6 \mathrm{H}), 3.86(\mathrm{~s}, 3 \mathrm{H}), 3.74(\mathrm{~s}$, $3 \mathrm{H}), 2.40(\mathrm{~s}, 3 \mathrm{H}) ;{ }^{13} \mathrm{C}$ NMR (101 MHz, DMSO- $\left.d_{6}\right) \delta$ : $172.60,167.19,164.35,161.12,160.58,158.48,157.24$, $153.03,151.75,139.86,139.65,139.22,132.70,131.23$, $129.69,126.58,125.43,123.49,108.39,106.26,96.44$, 93.46, 70.52, 60.46, 56.47, 56.43, 56.40, 21.15; HRMS calcd for $\mathrm{C}_{31} \mathrm{H}_{30} \mathrm{~N}_{3} \mathrm{O}_{10}[\mathrm{M}+\mathrm{H}]^{+}$604.1925, found 604.1919.

2-[5,7-二甲氧基-4-酮-2-(3,4,5-三甲氧基苯基)- $4 H$ 色烯-3-基]氧基- $N$-[5-(3,4-二氯苯基)-1,3,4-噁二唑-2-基] 乙酰胺 (4k): 黄色固体，产率 27.6\%. m.p. 220.1 $220.3{ }^{\circ} \mathrm{C} ;{ }^{1} \mathrm{H}$ NMR (400 MHz, $\left.\mathrm{CDCl}_{3}\right) \delta: 12.95$ (s, 1H), $8.19(\mathrm{~d}, J=1.9 \mathrm{~Hz}, 1 \mathrm{H}), 7.95(\mathrm{dd}, J=8.4,2.0 \mathrm{~Hz}, 1 \mathrm{H})$, $7.58(\mathrm{~d}, J=8.4 \mathrm{~Hz}, 1 \mathrm{H}), 7.26(\mathrm{~s}, 2 \mathrm{H}), 6.58(\mathrm{~d}, J=2.1 \mathrm{~Hz}$, $1 \mathrm{H}), 6.44(\mathrm{~d}, J=2.1 \mathrm{~Hz}, 1 \mathrm{H}), 4.41(\mathrm{~s}, 2 \mathrm{H}), 4.03(\mathrm{~s}, 3 \mathrm{H})$, 3.97 (s, 3H), $3.96(\mathrm{~s}, 6 \mathrm{H}), 3.95(\mathrm{~s}, 3 \mathrm{H}) ;{ }^{13} \mathrm{C}$ NMR (101 $\left.\mathrm{MHz}, \mathrm{CDCl}_{3}\right) \delta: 174.95,166.78,165.21,161.09,159.56$, $159.12,157.34,154.68,153.59,141.20,141.00,135.82$, $133.55,131.15,128.38,125.78,124.37,123.58,108.35$, 105.81, 96.64, 92.76, 73.54, 61.15, 56.66, 56.53, 56.06; HRMS calcd for $\mathrm{C}_{30} \mathrm{H}_{26} \mathrm{Cl}_{2} \mathrm{~N}_{3} \mathrm{O}_{10}[\mathrm{M}+\mathrm{H}]^{+} 658.0989$, found 658.0977 .

2-[5,7-二甲氧基-4-酮-2-(3,4,5-三甲氧基苯基)- $4 H$ 色烯-3-基]氧基- $N$-[5-(4-溴苯基)-1,3,4-噁二唑-2-基]乙酰 胺(41): 白色固体，产率 30.0\%. m.p. 243.1 247.7 ${ }^{\circ} \mathrm{C} ;{ }^{1} \mathrm{H}$ NMR (400 MHz, DMSO- $\left.d_{6}\right) \delta: 12.17(\mathrm{~s}, 1 \mathrm{H}), 7.87 \sim 7.80$ $(\mathrm{m}, 4 \mathrm{H}), 7.48(\mathrm{~s}, 2 \mathrm{H}), 6.90(\mathrm{~s}, 1 \mathrm{H}), 6.53(\mathrm{~s}, 1 \mathrm{H}), 4.86(\mathrm{~s}$, 2H), 3.92 (s, 3H), 3.89 (s, 6H), 3.87 (s, 3H), 3.75 (s, 3H); ${ }^{13} \mathrm{C}$ NMR (101 MHz, DMSO- $\left.d_{6}\right) \delta: 172.78,167.28$, $164.53,160.78,160.50,158.65,157.66,153.21,151.93$, $140.10,139.82,133.03,128.35,125.69,125.59,123.00$, 108.59, 106.50, 96.61, 93.65, 70.71, 60.64, 56.65, 56.60; HRMS calcd for $\mathrm{C}_{30} \mathrm{H}_{27} \mathrm{BrN}_{3} \mathrm{O}_{10}[\mathrm{M}+\mathrm{H}]^{+}$668.0874, found 668.0863 .

2-[5,7-二甲氧基-4-酮-2-(3,4,5-三甲氧基苯基)-4H色烯-3-基]氧基- $N$-[5-(2,4-二氯苯基)-1,3,4-噁二唑-2-基] 乙酰胺 $(4 \mathrm{~m})$ : 白色固体, 产率 24.5\%. m.p. 228.7 $229.3{ }^{\circ} \mathrm{C}$; ${ }^{1} \mathrm{H}$ NMR (400 MHz, DMSO- $\left.d_{6}\right) \delta$ : 12.26 (s, 1H), $7.96(\mathrm{~s}, 1 \mathrm{H}), 7.93$ (d, $J=1.2 \mathrm{~Hz}, 1 \mathrm{H}), 7.70$ (dd, $J=$ 8.5, $2.1 \mathrm{~Hz}, 1 \mathrm{H}), 7.50(\mathrm{~s}, 2 \mathrm{H}), 6.92(\mathrm{~d}, J=2.2 \mathrm{~Hz}, 1 \mathrm{H})$, $6.55(\mathrm{~d}, J=2.2 \mathrm{~Hz}, 1 \mathrm{H}), 4.88(\mathrm{~s}, 2 \mathrm{H}), 3.94(\mathrm{~s}, 3 \mathrm{H}), 3.90(\mathrm{~s}$, $6 \mathrm{H}), 3.88(\mathrm{~s}, 3 \mathrm{H}), 3.77(\mathrm{~s}, 3 \mathrm{H}) ;{ }^{13} \mathrm{C} \mathrm{NMR}(101 \mathrm{MHz}$, DMSO- $\left.d_{6}\right) \delta: 172.78,167.23,164.54,160.79,158.65$, 
$158.43,157.97,153.21,151.92,140.12,139.82,137.33$, $133.12,132.51,131.20,128.71,125.59,121.98,108.59$, 106.52, 96.62, 93.66, 70.72, 60.64, 56.65, 56.61; HRMS calcd for $\mathrm{C}_{30} \mathrm{H}_{26} \mathrm{Cl}_{2} \mathrm{~N}_{3} \mathrm{O}_{10}[\mathrm{M}+\mathrm{H}]^{+} 658.0989$, found 658.0984 .

2-[5,7-二甲氧基-4-酮-2-(3,4,5-三甲氧基苯基)-4H色烯-3-基]氧基- $N$-[5-(3,4-二甲氧基苯基)-1,3,4-噁二唑2-基]乙酰胺(4n): 白色固体, 产率 27.4\%. m.p. 247.3 $237.7{ }^{\circ} \mathrm{C} ;{ }^{1} \mathrm{H}$ NMR $\left(400 \mathrm{MHz}, \mathrm{CDCl}_{3}\right) \delta: 12.51(\mathrm{~s}, 1 \mathrm{H})$, $7.68(\mathrm{dd}, J=8.4,1.9 \mathrm{~Hz}, 1 \mathrm{H}), 7.63(\mathrm{~d}, J=1.8 \mathrm{~Hz}, 1 \mathrm{H})$, 7.29 (s, 2H), 6.97 (d, $J=8.5 \mathrm{~Hz}, 1 \mathrm{H}), 6.59$ (d, $J=2.1 \mathrm{~Hz}$, $1 \mathrm{H}), 6.45(\mathrm{~d}, J=2.1 \mathrm{~Hz}, 1 \mathrm{H}), 4.44(\mathrm{~s}, 2 \mathrm{H}), 4.03(\mathrm{~s}, 3 \mathrm{H})$, 4.00 (s, 3H), 3.98 (d, $J=3.3 \mathrm{~Hz}, 12 \mathrm{H}), 3.96(\mathrm{~s}, 3 \mathrm{H}) ;{ }^{13} \mathrm{C}$ NMR $\left(101 \mathrm{MHz}, \mathrm{CDCl}_{3}\right) \delta: 174.72,166.75,165.08$, $161.61,161.07,159.08,156.54,154.43,153.55,151.76$, $149.24,141.10,140.97,124.47,120.30,116.44,110.95$, 109.24, 108.46, 105.85, 96.56, 92.76, 73.37, 61.11, 56.61, $56.52,56.19,56.03$; HRMS calcd for $\mathrm{C}_{32} \mathrm{H}_{32} \mathrm{~N}_{3} \mathrm{O}_{12}[\mathrm{M}+$ $\mathrm{H}]^{+} 650.1980$, found 650.1978 .

2-[5,7-二甲氧基-4-酮-2-(3,4,5-三甲氧基苯基)-4H色烯-3-基]氧基- $N$-[5-(3-溴苯基)-1,3,4-啞二唑-2-基]乙酰 胺(4o): 白色固体, 产率 $32.3 \%$. m.p. $224.3 \sim 224.7{ }^{\circ} \mathrm{C}$; ${ }^{1} \mathrm{H}$ NMR $\left(400 \mathrm{MHz}, \mathrm{CDCl}_{3}\right) \delta: 12.86(\mathrm{~s}, 1 \mathrm{H}), 8.18(\mathrm{~d}, J=$ $1.9 \mathrm{~Hz}, 1 \mathrm{H}), 7.94(\mathrm{dd}, J=8.4,2.0 \mathrm{~Hz}, 1 \mathrm{H}), 7.58$ (d, $J=8.4$ $\mathrm{Hz}, 1 \mathrm{H}), 7.26$ (s, 3H), $6.58(\mathrm{~d}, J=2.1 \mathrm{~Hz}, 1 \mathrm{H}), 6.43$ (d, $J=$ $2.1 \mathrm{~Hz}, 1 \mathrm{H}), 4.41$ (s, 2H), 4.02 (s, 3H), 3.97 (s, 3H), 3.96 (s, 6H), 3.95 (s, 3H); ${ }^{13} \mathrm{C}$ NMR $\left(101 \mathrm{MHz}, \mathrm{CDCl}_{3}\right) \delta$ : 174.86, 166.74, 165.17, 161.09, 159.53, 159.10, 157.30, $154.58,153.58,141.16,141.05,135.77,133.52,131.12$, $128.35,125.75,124.37,123.62,108.38,105.88,96.61$, 92.77, 73.47, 61.12, 56.63, 56.53, 56.04; HRMS calcd for $\mathrm{C}_{30} \mathrm{H}_{27} \mathrm{BrN}_{3} \mathrm{O}_{10}[\mathrm{M}+\mathrm{H}]^{+}$668.0874, found 668.0861.

2-[5,7-二甲氧基-4-酮-2-(3,4,5-三甲氧基苯基)- $4 \mathrm{H}$ 色烯-3-基]氧基- $N$-[5-(2-溴苯基)-1,3,4-啞二唑-2-基]乙酰 胺(4p): 白色固体, 产率 38.5\%. m.p. 209.9 210.3 ${ }^{\circ} \mathrm{C}$; ${ }^{1} \mathrm{H}$ NMR (400 MHz, DMSO- $d_{6}$ ) $\delta$ : $12.21(\mathrm{~s}, 1 \mathrm{H}), 8.02$ (s, $1 \mathrm{H}), 7.88(\mathrm{~d}, J=33.0 \mathrm{~Hz}, 2 \mathrm{H}), 7.58(\mathrm{~s}, 1 \mathrm{H}), 7.48(\mathrm{~s}, 2 \mathrm{H})$, $6.92(\mathrm{~s}, 1 \mathrm{H}), 6.54(\mathrm{~s}, 1 \mathrm{H}), 4.85(\mathrm{~s}, 2 \mathrm{H}), 3.89(\mathrm{~s}, 12 \mathrm{H}), 3.74$ $(\mathrm{s}, 3 \mathrm{H}) ;{ }^{13} \mathrm{C}$ NMR $\left(101 \mathrm{MHz}\right.$, DMSO- $\left.d_{6}\right) \delta: 172.78$, $167.27,164.53,160.76,159.77,158.65,157.79,153.20$, $151.93,140.06,139.83,134.85,132.23,128.73,125.92$, $125.60,125.48,122.84,108.57,106.46,96.62,93.65$, $70.74,60.64,56.65,56.58$; HRMS calcd for $\mathrm{C}_{30} \mathrm{H}_{27} \mathrm{BrN}_{3} \mathrm{O}_{10}[\mathrm{M}+\mathrm{H}]^{+} 668.0874$, found 668.0862 .
3.3 目标化合物 $4 \mathrm{j}$ 与南方水稻黑条矮缩病毒 P $9-1$ 的微 量热涌动实验

取 $75 \mu \mathrm{L}$ 已纯化的 SRBSDV P9-1 蛋白(PDB 号: $5 \mathrm{EFT})^{[2]}$, 向其中加入 $1 \mu \mathrm{L}$ NHS-647 染料, 黑暗条件下 孵育 $30 \mathrm{~min}$; 将已标记的蛋白装入洗脱柱, 用磷酸缓冲 液(phosphate buffer saline, PBS)进行洗脱并收集蛋白. 分别向 16 个 PCR 管中加入 $10 \mu \mathrm{L}$ 梯度稀释(分别为 500 、 250、125、62.5、31.25、15.625、7.813、3.906、1.953、 $0.977 、 0.488 、 0.244 、 0.122 、 0.061 、 0.0305 、 0.01525$ $\mu \mathrm{mol} / \mathrm{L}$ )的化合物 $\mathbf{j}$, 然后将 $10 \mu \mathrm{L}$ 的已标记的蛋白和 10 $\mu \mathrm{L}$ 已稀释的分子混合, $5 \mathrm{~min}$ 孵育后，将每管复合物装 载到毛细吸管之中, 然后放置到毛细吸管的托盘上, 使 用 NT.115 仪器测量.

\section{4 目标化合物的抑菌活性测试}

通过浊度法, 用商品化的叶枯唑(Bismerthiazol)为 阳性对照药剂, 以 Xoo 和 Xac 对目标化合物进行抑菌活 性测试.

配制无菌营养肉汤培养基: $3.0 \mathrm{~g}$ 牛肉提取物, $5.0 \mathrm{~g}$ 蛋白胨, $1.0 \mathrm{~g}$ 酵母粉, $10.0 \mathrm{~g}$ 葡萄糖和 $1000 \mathrm{~mL}$ 二次水, pH 7.0 7.2.

在试管中将待测化合物和对照药剂配制成 100 和 $50 \mu \mathrm{g} / \mathrm{mL}$ 浓度的培养基, 测试其 OD 值, 该值即为无菌 培养基的 $\mathrm{OD}$ 值. 将 $40 \mu \mathrm{L}$ 含菌的肉汤培养基加入到无 菌培养基中, 将接菌的试管在 $28{ }^{\circ} \mathrm{C}$ 下以 $180 \mathrm{r} / \mathrm{min}$ 连续 摇动捊育 36 48 h, 然后测其 OD 值. 以下列公式计算 抑制率:

\section{抑制率 $(\%)=($ 校正后对照培养基菌液 OD 值一 \\ 校正后含毒培养基 OD 值)/校正后对照培养基菌液 OD 值 $\times 100 \%$ \\ 校正 OD 值=含菌培养基 OD 值一无菌培养基 OD 值}

\section{5 目标化合物的抗 $T M V$ 活性测试}

\subsection{1 目标化合物对 $T M V$ 的治疗活性测试}

取一株心叶烟, 将其修剪保留 3 5 片烟叶, 在每片 叶子上均匀地撒上金刚砂，用排笔蘸取 $T M V$ 病毒 $(500$ $\mu \mathrm{g} / \mathrm{mL})$, 均匀地刷在烟叶上. 待病毒接种上烟叶 $(0.5 \sim 1$ h), 用清水洗去金刚砂，自然晾干后，用毛笔蘸取目标 化合物溶剂 $(500 \mathrm{mg} / \mathrm{mL})$ 均匀地涂抹在烟叶的右半叶. 晾干后, 将其移至 $28{ }^{\circ} \mathrm{C}$ 条件下保湿培养. 2 3 d 后, 叶 片出现明显的斑点, 记录左右两边的斑点数, 带入公式 计算其抑制率. 每个化合物平行进行 2 3 次.

\subsection{2 目标化合物对 $T M V$ 的保护活性测试}

取一株心叶烟, 将其修剪保留 3 5 片烟叶, 用毛笔 蘸取目标化合物溶剂 $(500 \mathrm{mg} / \mathrm{mL})$ 均匀地涂抹在烟叶的 
右半叶. $12 \mathrm{~h}$ 后, 将金刚砂均匀地撒在叶片的左右两边, 用排笔蘸取 $T M V$ 病毒 $(500 \mu \mathrm{g} / \mathrm{mL})$, 均匀地刷在烟叶上. 待病毒接种上烟叶 $(0.5 \sim 1 \mathrm{~h})$, 用清水洗去金刚砂, 自然 晾干后, 移至 $28{ }^{\circ} \mathrm{C}$ 条件下保湿培养. $2 \sim 3 \mathrm{~d}$ 后, 叶片出 现明显的斑点, 记录左右两边的斑点数, 带入公式计算 其抑制率. 每个化合物平行进行 2 3 次.

\subsection{3 抗 $T M V$ 的抑制率计算}

2 3 d 后观察叶片, 当叶片上出现明显枯斑, 以同 叶左半叶作为空白对照, 宁南霉素作为药剂对照, 记录 下每叶片左右斑点数, 如下公式计算其抗 $T M V$ 活性的 抑制率.

$$
I=(L-R) / L \times 100 \%
$$

其中, $I$ 为目标化合物抗 $T M V$ 活性的抑制率; $L$ 为左 半叶的枯斑数, $R$ 为右半叶的枯斑数.

辅助材料(Supporting Information) 文中所有化合物 的核磁共振谱图以及目标化合物的 HRMS. 这些材料可 以免费从本刊网站(http://sioc-journal.cn/)上下载.

\section{References}

[1] Bos, L. Trends Microbiol. 2000, 8, 82.

[2] Li, X. Y.; Liu, J.; Yang, X.; Ding, Y.; Wu, J.; Hu, D. Y.; Song, B. A. Bioorg. Med. Chem. Lett. 2015, 23, 3629.

[3] Zhou, G. H.; Wen, J. J.; Cai, D. J.; Li, P.; Xu, D. L.; Zhang, S. G. Chin. Sci. Bull. 2008, 53, 3677.

[4] Huang, N.; Angeles, E. R.; Domingo, J.; Magpanty, G.; Singh, S.; Zhan, G.; Kumarvadivel, N.; Bennett, J.; Khush, G. S. Theor. Appl. Genet. 1997, 95, 313.

[5] Guo, R.-X.; Li, L.-G.; Wang, Y.-F.; Huo, C.-H.; Fu, Y.; Wang, L.; Shi, W.-Q. Chin. Tradit. Herbal Drugs 2015, 46, 2019 (in Chinese). (郭瑞霞, 李力更, 王于方, 霍长虹, 付炎, 王磊, 史文清, 中草 药, 2015, 46, 2019.)

[6] Liu, C.-L.; Li, Z.-M. Pesticides 2003, 42, 1 (in Chinese). (刘长令, 李正名, 农药, 2003, 42, 1.)

[7] Mei, Q.-G.; Yuan, W.-C.; Wang, C. Chin. J. Org. Chem. 2015, 35, 70 (in Chinese).

(梅青刚, 袁伟成, 王淳, 有机化学, 2015, 35, 70.)
[8] Yu, M. S.; Lee, J.; Lee, J. M.; Kim, Y.; Chin, Y. W.; Jee, J. G.; Keum, Y. S.; Jeong, Y. J. Bioorg. Med. Chem. Let. 2012, 22, 4049.

[9] Nguyen, T. H. V.; Trinh, A. V.; Nguyen, X. N.; Kiem, P. V.; Minh, C. V.; Long, P. Q.; Anh, L.T.; Cuong, N. M.; Song, J. H.; Ko, H. J.; Kim, N, Park, S. J.; Kim, S. H. Nat. Prod. Commun. 2014, 9, 643.

[10] Zhong, X. M.; Wang, X. B.; Chen, L. J.; Ruan, X. H.; Li, Q.; Zhang, J. P.; Chen, Z.; Xue, W. Chem. Cent. J. 2017, 11, 106.

[11] Chen, C. C; Huang, C. Y. Protein J. 2011, 30, 59.

[12] Rashed, K.; Ćirić, A.; Glamočlija, J.; Sokovic, M. Ind. Crop. Prod. 2014, 59, 210.

[13] Xiao, W.; Ruan, X.-H.; Li, Q.; Zhang, J.-P.; Zhong, X.-M.; Xie, Y.; Wang, X.-B.; Huang, M.-G.; Xue, W. Chem. J. Chin. Univ. 2017, 38, 35 (in Chinese).

(肖维, 阮祥辉, 李琴, 张菊平, 钟新敏, 谢艳, 王晓斌, 黄民国, 薛伟, 高等学校化学学报, 2017, 38, 35.)

[14] Chobot, V.; Hadacek, F. Redox Rep. 2011, 16, 242.

[15] Zhao, L.; Xu, S. P.; Li, Z. Y.; Zhang, L.; Zhang, Z. S.; Pan, R. L. Sci. Technol. Food Ind. 2012, 33, 56.

[16] Xue, W.; Song, B. A.; Zhao, H. J. Eur. J. Med. Chem. 2015, 97, 155.

[17] Tae, K. H.; Inae, J.; Mi, E. K.; Bae, S. K.; Lee, J. K. Biomed. Pharmacother. 2017, 91, 378.

[18] Huang, M.-G.; Ruan, X.-H.; Zhang, J.-P.; Li, Q.; Wang, Y.-H.; Chen, L.-J.; Zhang, C.; Li, P. Chin. J. Org. Chem. 2017, 37, 2145 (in Chinese). (黄民国，阮祥辉，张菊平，李琴，王一会，陈丽娟，张橙，李普， 有机化学, 2017, 37, 2145.)

[19] Ningaiah, S.; Bhadraiah, U. K.; Doddaramappa, S. D.; Keshavamurthy, K. Bioorg. Med. Chem. Lett. 2014, 24, 245.

[20] Li, P.; Shi, L.; Gao, M. L.; Yang, X.; Xue, W.; Jin, L. H.; Hu, D. Y.; Song, B. A. Bioorg. Med. Chem. Lett. 2015, 25, 481.

[21] Gan, X. H.; Hu, D. Y.; Chen, Z.; Wang, Y. J.; Song, B. A. Bioorg. Med. Chem. Lett. 2017, 27, 4298.

[22] Wu, W. N; Chen, Q.; Tai, A. Q.; Jiang, G. Q.; Ouyang, G. P. Bioorg. Med. Chem. Lett. 2015, 25, 2243.

[23] Ragab, F. A. F.; Abou-Seri, S. M.; Abdel-Aziz, S. A.; Alfayomy, A. M; Aboelmagd, M. Eur. J. Med. Chem. 2017, 138, 140

[24] Zhang, S.; Luo, Y.; He, L. Q.; Liu, Z. J.; Jiang, A. Q.; Yang, Y. H.; Zhu, H. L. Bioorg. Med. Chem. Lett. 2013, 21, 3723.

[25] Aziz-Ur-Rehman; Siddiqui, S. Z.; Abbasi, M. A.; Abbas, N.; Khan, K. M.; Shahid, M.; Mahmood, Y. Int. J. Pharm. Pharm. Sci. 2012, 4, 676 .

[26] Rajak, H.; Kharya, M. D.; Mishra, P. Arch. Pharm. Chem. Life Sci. 2008, 341, 247.

[27] Niu, P. F.; Kang, J. F.; Tian, X. H.; Song, L. N.; Liu, H. X.;Wu, J.; Yu, W. Q.; Chang, J. B. J. Org. Chem. 2015, 80, 1018. 